\title{
TRANSIENT ANALYSIS OF A DISCOURAGED ARRIVAL QUEUE SUBJECT TO TOTAL CATASTROPHES, FAILURES AND REPAIRS
}

\author{
S. Sophia \\ Department of Mathematics \\ SSN College of Engineering \\ SSN Nagar, Kalavakkam-603 110, TamilNadu, INDIA
}

\begin{abstract}
In this paper, a single server discouraged arrival queue subject to catastrophes, server failures and non-zero repair time is considered. Explicit expressions for the transient probabilities of system size are obtained using continued fractions technique. The corresponding steady state probabilities are deduced. Some important system performance measures are discussed. Further, reliability and availability of the system are analyzed.
\end{abstract}

AMS Subject Classification: 60K25

Key Words: discouraged arrivals, catastrophes, server failure, continued fractions, reliability analysis

\section{Introduction}

Queueing systems have been extensively studied by many authors and they constitute a central tool in modeling and performance analysis of computer systems, communication networks, machine plants, air traffic, manufacturing systems and so forth. Stochastic simulations are only capable of producing estimates and modeling large systems is a very cumbersome process. Simulation results do not accurately portray the system behavior in a vast majority of the cases. Therefore, it is important to have an analytical tractable model and numerical techniques to analyze the queueing systems.

Received: March 11, 2016

Published: June 1, 2016
(C) 2016 Academic Publications, Ltd.

url: www.acadpubl.eu 
Despite the enormous literature on queueing theory, a very few stochastic systems are known to have closed-form transient solutions for the distribution of the process. Studying the steady-state behavior of the system is simple and straightforward techniques can be employed. Moreover, stationary results are mainly used within the system design process and it cannot give insight into the transient behavior of the system. Transient analytical results can be used directly to study the finite time properties and they are useful in obtaining optimal solutions which lead to the control of the queueing system. For instance, in the evaluation of high-speed broad band networks new performance measures such as interval-based quality of service measures require transient analysis (see Nagarajan and Kurose [11]). Researchers used different techniques to derive the transient solution of queueing systems, in particular Bailey [3] used generating function method, Champernowne [4] used combinatorial method, difference equation was used by Conolly [5] where as Parthasarathy and Sudhesh [15] used continued fraction technique to study the transient behavior.

In many practical situations, the service facility possesses defence mechanisms against long waiting lines. For instance, the congestion control mechanism prevents the formation of long queues in computer and communication systems by controlling the transmission rates of packets based on the queue length(of packets) at source or destination. Moreover, a long waiting line may force the servers to increase their rate of service as well as discourage prospective customers which results in balking. Hence, it is of interest to study queueing systems taking into consideration the state-dependent nature of the system. In state-dependent queues the arrival and service rates depend on the number of customers in the system. Getting analytical results for time-dependent analysis of the state-dependent queueing systems is usually difficult and often impossible. In spite of its difficulty, Parthasarathy and Sudhesh [16] provided the transient solution of state-dependent birth death process. Krishna kumar et al [9] discussed a Markovian queue with chain sequence rates and total catastrophes.

The notion of catastrophes was introduced by Gelenbe [6] and subsequent work has been carried out by many researchers. Practical queueing systems like computer, communication networks, neural networks and manufacturing systems are not reliable and disasters may occur in them, thereby violating the operation of the system and in particular leading to loss of several or all customers. In a computer network, negative customers(catastrophes) can represent viruses, in a neural network, negative and positive customers can represent inhibitory and excitatory signals respectively and in manufacturing systems, negative customers can represent orders of demand. Catastrophes in a queueing system can 
also be considered as a type of clearing mechanism which removes all the work load in the system whenever it occurs. One can think of a catastrophe as a server reset or unplug which causes all the jobs in the system to be last. For a comprehensive analysis of queueing networks with catastrophes readers may refer to Tarabia [20] and Noam Paz and Yechiali [18].

Queueing models with server breakdowns are more realistic in computer and communication switching systems since the failure and repair of processors have a major impact on the flow of jobs that have to be handled by those processors (Towsley and Tripathi [21] and Wartenhorst [23]). Such repairable queueing models are interesting, either from the point of view of queueing theory or of reliability theory. Krishna kumar and Pavai Madheswari [8] determined the transient solution for an $M / M / 1$ queue with breakdown and repairable server subject to catastrophes and discussed the reliability and availability of the system.

In this paper, a catastrophic queueing model with discouraged arrivals along with server break down and repair is considered. Transient solutions of the state-dependent queueing model with the above mentioned features are obtained using continued fractions. The rest of the paper is organized as follows: The model under discussion is described in Section 2. Section 3 gives a detailed analysis of the transient state probabilities using continued fractions. Corresponding steady state probabilities, some important performance measures and the availability and reliability of the system under consideration are discussed in Section 4. The appendix provides some relations and identities involving confluent hypergeometric functions and continued fractions which are being used in this paper.

\section{Model Description}

The system under study can be described in the following way. We consider a single server queueing system with state-dependent arrival and service rates, subject to catastrophes at the service station. There is infinite room for customers to wait. Customers are served on a first-come, first-served basis. Let $\left\{X(t), t \in R^{+}\right\}$be the number of customers in the system at time $t$. We assume that the arrival and service rates are $\lambda_{n}$ and $\mu_{n}$ respectively where the number of customers in the system at time $t$ is $n$; in any small interval $(t, t+\triangle t), \triangle t \geq 0$ an arrival occurs with probability $\lambda_{n} \triangle t+o(\triangle t)$; a service being completed with probability $\mu_{n} \triangle t+o(\triangle t)$. It is obvious that in this interval neither an arrival nor service takes place with probability $1-\left(\lambda_{n}+\mu_{n}\right) \triangle t+o(\triangle t)$. 
Apart from arrival and service processes, the catastrophes also occur at the service facility as a Poisson process with rate $\alpha$, when the server is idle or busy serving the customers (i.e.,the server is operational). Whenever a catastrophe occurs at the busy server, all the customers in the system are wiped out instantly, the server gets inactivated and is sent for repair. The catastrophes may come either from outside the system or from another service station. The repair times of failed server are i.i.d, according to an exponential distribution with mean $\frac{1}{\eta}$. After the completion of repair, the server immediately returns to its operational state and is ready for service when a new customer arrives. In addition, it is assumed that the newly arriving customers during the repair time of failed server will be lost forever.

Let $P_{n}(t)=P(X(t)=n), n=0,1,2, \ldots$, denote the transient probabilities that there are $n$ customers in the system at time $t$, when the server is in operational state. $Q(t)$ be the probability that the server is under repair at time $t$.

Based on the above assumptions, the state probabilities $P_{n}(t)$, $n=0,1,2, \ldots$, and the failure probability $Q(t)$ can be described by the differential-difference equations governing the system as follows:

$$
\begin{gathered}
\frac{d Q(t)}{d t}=-\eta Q(t)+\alpha[1-Q(t)] \\
\frac{d P_{0}(t)}{d t}=-\left(\alpha+\lambda_{0}\right) P_{0}(t)+\mu_{1} P_{1}(t)+\eta Q(t) \\
\frac{d P_{n}(t)}{d t}=-\left(\begin{array}{r}
\left.\lambda_{n}+\mu_{n}+\alpha\right) P_{n}(t)+\lambda_{n-1} P_{n-1}(t) \\
+\mu_{n+1} P_{n+1}(t), \quad n=1,2,3, \ldots
\end{array}\right.
\end{gathered}
$$

Without loss of generality, assume that initially there is no customer in the system, so that $P_{0}(0)=1 \quad \& \quad Q(0)=0$.

\section{Discouraged Arrivals}

Queues with discouraged arrivals have applications in computers with batch job processing (see Ng chee Hock [13]). Natvig [12] obtained transient solutions for these state-dependent queues. Sophia and Vijayakumar [19] and Parthasarathy and Vijayashree [17] considered fluid queues with discouraged arrivals and obtained explicit expressions for the buffer content distribution. Job submissions 
are discouraged when the facility is frequently used and are modeled as a Poisson process with sate-dependent arrivals. The time taken to process each job is exponentially distributed with a constant service rate, regardless of the number of jobs in the system. For the system under consideration, the arrival and service rates, when the system size is $n$, are given by

$$
\lambda_{n}=\frac{\lambda}{n+1}, \quad n=0,1,2, \ldots \quad \& \quad \mu_{n}=\mu, \quad n=1,2,3, \ldots
$$

In the following sequel,we denote by $g^{*}(s)$ as the Laplace transform of $g($.$) . By$ taking Laplace transforms and applying appropriate arrival and service rates as in (3.1) with the initial conditions, the above system of equations are reduced to a system of simultaneous equations given by

$$
\begin{gathered}
Q^{*}(s)=\frac{\alpha}{s(s+\eta+\alpha)} \\
(s+\lambda+\alpha) P_{0}^{*}(s)=1+\mu P_{1}^{*}(s)+\eta Q^{*}(s)
\end{gathered}
$$

and

$$
\left(s+\frac{\lambda}{n+1}+\mu+\alpha\right) P_{n}^{*}(s)=\frac{\lambda}{n} P_{n-1}^{*}(s)+\mu P_{n+1}^{*}(s), n=1,2,3, \ldots .
$$

From (3.2) and (3.3) after some algebra we get

$$
P_{0}^{*}(s)=\frac{1+\frac{\eta \alpha}{s(s+\eta+\alpha)}}{(s+\lambda+\alpha)-\mu \frac{P_{1}^{*}(s)}{P_{0}^{*}(s)}}
$$

and (3.4) gives

$$
\frac{P_{n}^{*}(s)}{P_{n-1}^{*}(s)}=\frac{\frac{\lambda}{n}}{\left(s+\frac{\lambda}{n+1}+\mu+\alpha\right)-\mu \frac{P_{n+1}^{*}(s)}{P_{n}^{*}(s)}}, \quad n=1,2,3, \ldots
$$

Now using (3.6) iteratively in (3.5), we get $P_{0}^{*}(s)$ as a continued fraction expression as (see Wall [22])

$$
P_{0}^{*}(s)=\frac{1+\frac{\eta \alpha}{s(s+\eta+\alpha)}}{(s+\lambda+\alpha)-} \frac{\lambda \mu}{\left(s+\frac{\lambda}{2}+\mu+\alpha\right)-} \frac{\frac{\lambda}{2} \mu}{\left(s+\frac{\lambda}{3}+\mu+\alpha\right)-} \ldots
$$

Now, making use of the identity (A.6) of Appendix, the above equation can be expressed as

$$
P_{0}^{*}(s)=\left(1+\frac{\eta \alpha}{s(s+\eta+\alpha)}\right)\{s+\lambda+\alpha+(s+\alpha+\mu)
$$




$$
\begin{aligned}
\times & {\left[\left(\frac{\lambda(s+\alpha)}{(s+\mu+\alpha)^{2}}+1\right) \frac{{ }_{1} F_{1}\left(1 ; \frac{\lambda(s+\alpha)}{(s+\mu+\alpha)^{2}}+1 ; \frac{-\lambda \mu}{(s+\mu+\alpha)^{2}}\right)}{{ }_{1} F_{1}\left(2 ; \frac{\lambda(s+\alpha)}{(s+\mu+\alpha)^{2}}+2 ; \frac{-\lambda \mu}{(s+\mu+\alpha)^{2}}\right)}\right.} \\
& \left.\left.-\left(\frac{\lambda+s+\mu+\alpha}{s+\mu+\alpha}\right)\right]\right\}^{-1}
\end{aligned}
$$

so that

$$
\begin{aligned}
P_{0}^{*}(s) & =\left(1+\frac{\eta \alpha}{s(s+\eta+\alpha)}\right)\left[(s+\mu+\alpha)\left(\frac{\lambda(s+\alpha)}{(s+\mu+\alpha)^{2}}+1\right)\right. \\
& \left.\times \frac{{ }_{1} F_{1}\left(1 ; \frac{\lambda(s+\alpha)}{(s+\mu+\alpha)^{2}}+1 ; \frac{-\lambda \mu}{(s+\mu+\alpha)^{2}}\right)}{{ }_{1} F_{1}\left(2 ; \frac{\lambda(s+\alpha)}{(s+\mu+\alpha)^{2}}+2 ; \frac{-\lambda \mu}{(s+\mu+\alpha)^{2}}\right)}-\mu\right] .
\end{aligned}
$$

Again, using the identity $(\mathrm{A} .4)$ and the fact ${ }_{1} F_{1}(0 ; c ; z)=1$ in $(3.8), P_{0}^{*}(s)$ simplifies to

$$
P_{0}^{*}(s)=\left(1+\frac{\eta \alpha}{s(s+\eta+\alpha)}\right) \frac{{ }_{1} F_{1}\left(2 ; \frac{\lambda(s+\alpha)}{(s+\mu+\alpha)^{2}}+2 ; \frac{-\lambda \mu}{(s+\mu+\alpha)^{2}}\right)}{(s+\alpha)\left(\frac{\lambda(s+\alpha)}{(s+\mu+\alpha)^{2}}+1\right)} .
$$

Successive iteration of (3.6) yields

$$
\begin{aligned}
& \frac{P_{n}^{*}(s)}{P_{n-1}^{*}(s)}= \frac{\frac{\lambda}{n}}{\left(s+\frac{\lambda}{n+1}+\mu+\alpha\right)-} \frac{\frac{\lambda \mu}{n+1}}{\left(s+\frac{\lambda}{n+2}+\mu+\alpha\right)-} \\
& \frac{\frac{\lambda \mu}{n+2}}{\left(s+\frac{\lambda}{n+3}+\mu+\alpha\right)-} \cdots \quad n=1,2,3, \ldots
\end{aligned}
$$

As before, using the identity (A.6) in the above equation, we have, for $n=1,2,3, \ldots$,

$$
\frac{P_{n}^{*}(s)}{P_{n-1}^{*}(s)}=\frac{\frac{1}{n} \frac{\lambda(n+1)}{(s+\mu+\alpha)}}{\left(\frac{\lambda(s+\alpha)}{(s+\mu+\alpha)^{2}}+n+1\right)} \frac{{ }_{1} F_{1}\left(n+2 ; \frac{\lambda(s+\alpha)}{(s+\mu+\alpha)^{2}}+n+2 ; \frac{-\lambda \mu}{(s+\mu+\alpha)^{2}}\right)}{{ }_{1} F_{1}\left(n+1 ; \frac{\lambda(s+\alpha)}{(s+\mu+\alpha)^{2}}+n+1 ; \frac{-\lambda \mu}{(s+\mu+\alpha)^{2}}\right)} .
$$

Iterating the above equation, we get

$$
P_{n}^{*}(s)=P_{0}^{*}(s) \prod_{j=1}^{n} \frac{P_{j}^{*}(s)}{P_{j-1}^{*}(s)}
$$




$$
\begin{aligned}
& =\frac{\left(1+\frac{\eta \alpha}{s(s+\eta+\alpha)}\right)\left(\frac{n+1}{s+\alpha}\right)\left(\frac{\lambda}{s+\mu+\alpha}\right)^{n}}{\left(\frac{\lambda(s+\alpha)}{(s+\mu+\alpha)^{2}}+1\right)\left(\frac{\lambda(s+\alpha)}{(s+\mu+\alpha)^{2}}+2\right) \ldots\left(\frac{\lambda(s+\alpha)}{(s+\mu+\alpha)^{2}}+n+1\right)} \\
& \times \quad{ }_{1} F_{1}\left(n+2 ; \frac{\lambda(s+\alpha)}{(s+\mu+\alpha)^{2}}+n+2 ; \frac{-\lambda \mu}{(s+\mu+\alpha)^{2}}\right), n=0,1,2, \ldots
\end{aligned}
$$

After some simplification and using (A.1), the above equation becomes

$$
\begin{aligned}
P_{n}^{*}(s) & =\frac{\left(1+\frac{\eta \alpha}{s(s+\eta+\alpha)}\right)(n+1) \lambda^{n+1}(s+\mu+\alpha)^{2(n+1)}}{(s+\mu+\alpha)^{n}[(s+\alpha) \lambda]\left[(s+\alpha) \lambda+(s+\mu+\alpha)^{2}\right]\left[(s+\alpha) \lambda+2(s+\mu+\alpha)^{2}\right] \ldots} \\
& \left.\times \sum_{k=0}^{\infty} \frac{\left.(n+2) \lambda+(n+1)(s+\mu+\alpha)^{2}\right]}{\left(\frac{\lambda(s+\alpha)}{(s+\mu+\alpha+\alpha)^{2}}\right)^{k}}+n+2\right)_{k} k !
\end{aligned}
$$

where $(.)_{k}$ is the Pochhammer symbol as defined in (A.2).

On further simplification (3.13) modifies to

$$
\begin{aligned}
P_{n}^{*}(s)= & \left(1+\frac{\eta \alpha}{s(s+\eta+\alpha)}\right) \sum_{k=0}^{\infty} \frac{(-1)^{k} \lambda^{n+k+1} \mu^{k}}{k ! n !(s+\mu+\alpha)^{n+2 k}} \\
\times & \frac{(s+\mu+\alpha)^{2(n+k+1)}(n+k+1) !}{n+k+1}\left[(s+\alpha) \lambda+l(s+\mu+\alpha)^{2}\right]
\end{aligned}
$$

By employing the partial fraction expansion, the above equation can be expressed as

$$
\begin{aligned}
P_{n}^{*}(s) & =\left(1+\frac{\eta \alpha}{s(s+\eta+\alpha)}\right) \sum_{k=0}^{\infty} \frac{(-1)^{k} \lambda^{n+k+1}}{k ! n !} \mu^{k} \sum_{l=0}^{n+k+1}\left(\begin{array}{c}
n+k+1 \\
l
\end{array}\right)(-1)^{l} \\
& \times \frac{1}{(s+\mu+\alpha)^{n+2 k}\left[(s+\alpha) \lambda+l(s+\mu+\alpha)^{2}\right]}, \quad n=0,1,2, \ldots
\end{aligned}
$$

On inversion, (3.14) yields, for $n=0,1,2, \ldots$,

$$
P_{n}(t)=\sum_{k=0}^{\infty} \frac{(-1)^{k} \lambda^{n+k+1}}{k ! n !} \mu^{k} \sum_{l=0}^{n+k+1}\left(\begin{array}{c}
n+k+1 \\
l
\end{array}\right)(-1)^{l}\left[g_{n+2 k, l}(t)\right.
$$




$$
\left.+\frac{\eta \alpha}{\eta+\alpha} \int_{0}^{t} g_{n+2 k, l}(u)\left[1-e^{-(\eta+\alpha)(t-u)}\right] d u\right]
$$

where

$$
g_{n+2 k, l^{*}}(s)=\frac{1}{(s+\alpha+\mu)^{2 k+n}\left[\lambda(s+\alpha)+l(s+\alpha+\mu)^{2}\right]}
$$

and

$$
\begin{gathered}
g_{0,0}(t)=\frac{e^{-\alpha t}}{\lambda} \\
g_{0,1}(t)=\frac{e^{-\left(\frac{\lambda}{2}+\mu+\alpha\right) t}}{\sqrt{\frac{\lambda^{2}}{4}+\lambda \mu}} \sinh \left(\sqrt{\frac{\lambda^{2}}{4}+\lambda \mu}\right) t \\
g_{n+2 k, 0}(t)=\frac{e^{-\alpha t}}{\lambda(2 k+n-1) !} \int_{0}^{t} e^{-\mu y} y^{n+2 k-1} d y, \text { for } n+2 k>0
\end{gathered}
$$

and for $n+2 k, l \geq 1$,

$$
\begin{aligned}
g_{n+2 k, l}(t) & =\frac{e^{-\left(\frac{\lambda}{2 l}+\mu+\alpha\right) t}}{l(n+2 k-1) ! \sqrt{\frac{\lambda^{2}}{4 l^{2}}+\frac{\lambda \mu}{l}}} \\
& \times \int_{0}^{t} e^{\frac{\lambda}{2 l} y} y^{n+2 k-1} \sinh \left(\sqrt{\frac{\lambda^{2}}{4 l^{2}}+\frac{\lambda \mu}{l}}\right)(t-y) d y .
\end{aligned}
$$

The transient probability of the failure distribution is obtained from (2.1) as follows

$$
Q(t)=\frac{\alpha}{\eta+\alpha}\left[1-e^{-}(\eta+\alpha) t\right]
$$

Thus, (3.15)-(3.20) completely determine all the state probabilities $P_{n}(t), n=$ $0,1,2, \ldots$ and the failure probability $Q(t)$ of the system size for the model under consideration. One can observe that for $\alpha=0$ (3.15) coincides with Parthasarathy and Selvaraju [14]. 


\section{Steady State Probabilities}

In this section, the structure of the steady state distributions of the system size and failure distribution of the discouraged arrival queueing system subject to catastrophes, failures and non-zero repair time is discussed. The moments related to the steady state system size probabilities are obtained in terms of confluent hypergeometric functions.

By multiplying (3.2) by $s$ on both sides and taking the limit as $s \rightarrow 0$, one gets

$$
\lim _{s \rightarrow 0} s Q^{*}(s)=\lim _{s \rightarrow 0} s \frac{\alpha}{s(s+\eta+\alpha)} .
$$

By using Tauberian theorem, the steady state failure distribution $Q$ is obtained as

$$
Q=\frac{\alpha}{(\eta+\alpha)}
$$

Multiplying equation (3.12) by $s$ on both sides and taking limit as $s \rightarrow 0$, one has

$$
\begin{aligned}
\lim _{s \rightarrow 0} s P_{n}^{*}(s) & =\lim _{s \rightarrow 0} s \frac{\left(1+\frac{\eta \alpha}{s(s+\eta+\alpha)}\right)\left(\frac{n+1}{s+\alpha}\right)\left(\frac{\lambda}{s+\mu+\alpha}\right)^{n}}{\left(\frac{\lambda(s+\alpha)}{(s+\mu+\alpha)^{2}}+1\right)\left(\frac{\lambda(s+\alpha)}{(s+\mu+\alpha)^{2}}+2\right) \ldots\left(\frac{\lambda(s+\alpha)}{(s+\mu+\alpha)^{2}}+n+1\right)} \\
& \times{ }_{1} F_{1}\left(n+2 ; \frac{\lambda(s+\alpha)}{(s+\mu+\alpha)^{2}}+n+2 ; \frac{-\lambda \mu}{(s+\mu+\alpha)^{2}}\right), n=0,1,2, \ldots
\end{aligned}
$$

By application of Tauberian theorem, we get $P_{n}$ in terms of hyper-geometric function as follows

$$
\begin{array}{r}
P_{n}=\frac{\eta}{\eta+\alpha} \frac{(n+1)\left(\frac{\lambda}{\alpha+\mu}\right)^{n}{ }_{1} F_{1}\left(n+2 ; \frac{\lambda \alpha}{(\alpha+\mu)^{2}}+n+2 ; \frac{-\lambda \mu}{(\alpha+\mu)^{2}}\right)}{\left(\frac{\lambda \alpha}{(\alpha+\mu)^{2}}+1\right)\left(\frac{\lambda \alpha}{(\alpha+\mu)^{2}}+2\right) \ldots\left(\frac{\lambda \alpha}{(\alpha+\mu)^{2}}+n+1\right)} \\
n=0,1,2, \ldots
\end{array}
$$

and in particular

$$
P_{0}=(1-Q) \frac{{ }_{1} F_{1}\left(2 ; \frac{\lambda \alpha}{(\alpha+\mu)^{2}}+2 ; \frac{-\lambda \mu}{(\alpha+\mu)^{2}}\right)}{\frac{\lambda \alpha}{(\alpha+\mu)^{2}}+1}
$$


$P_{n}$ can also be obtained from (3.14) using Tauberian theorem as

$$
\begin{aligned}
P_{n} & =\frac{\eta \alpha}{\eta+\alpha} \sum_{k=0}^{\infty} \frac{(-1)^{k} \lambda^{n+k+1}}{k ! n !} \mu^{k} \sum_{l=0}^{n+k+1}\left(\begin{array}{c}
n+k+1 \\
l
\end{array}\right)(-1)^{l} \\
& \times \frac{1}{(\alpha+\mu)^{n+2 k}\left[\alpha \lambda+l(\alpha+\mu)^{2}\right]}, \quad n=0,1,2, \ldots
\end{aligned}
$$

For $\alpha=0,(4.4)$ reduces to

$$
P_{n}=\exp \left[\frac{-\lambda}{\mu}\right] \frac{\left(\frac{\lambda}{\mu}\right)^{n}}{n !}, \quad n=0,1,2, \ldots,
$$

a well known result of Gross and Harris [7]. Now, based on the above steady state probabilities the moments are determined using probability generating function. By multiplying (4.1) and (4.2) by $z^{n}$ and summing over $n$, steady state probability generating function $\prod(z)$ is obtained as

$$
\begin{aligned}
\prod(z) & =\frac{\alpha}{\eta+\alpha}+\frac{\eta}{\eta+\alpha} \sum_{n=0}^{\infty} \frac{(n+1)\left(\frac{\lambda z}{\alpha+\mu}\right)^{n}}{\left(\frac{\lambda \alpha}{(\alpha+\mu)^{2}}+1\right)\left(\frac{\lambda \alpha}{(\alpha+\mu)^{2}}+2\right) \ldots\left(\frac{\lambda \alpha}{(\alpha+\mu)^{2}}+n+1\right)} \\
& \times{ }_{1} F_{1}\left(n+2 ; \frac{\lambda \alpha}{(\alpha+\mu)^{2}}+n+2 ; \frac{-\lambda \mu}{(\alpha+\mu)^{2}}\right)
\end{aligned}
$$

Now using the identity (A.7), (4.6) modifies to

$$
\prod(z)=\frac{\alpha}{\eta+\alpha}+\frac{\eta}{(\eta+\alpha)} \frac{{ }_{1} F_{1}\left(2 ; \frac{\lambda \alpha}{(\alpha+\mu)^{2}}+2 ; \frac{-\lambda \mu}{(\alpha+\mu)^{2}}+\frac{\lambda z}{(\alpha+\mu)}\right)}{\left(\frac{\lambda \alpha}{(\alpha+\mu)^{2}}+1\right)} .
$$

Differentiation of (4.7) (refer (A.8)) with respect to $z$ and setting $z=1$ gives the steady state moments $E(X)$ and $E\left(X^{2}\right)$ as follows

$$
\begin{gathered}
E(X)=\frac{\eta}{(\eta+\alpha)} \frac{\frac{2 \lambda}{(\alpha+\mu)}{ }_{1} F_{1}\left(3 ; \frac{\lambda \alpha}{(\alpha+\mu)^{2}}+3 ; \frac{\lambda \alpha}{(\alpha+\mu)^{2}}\right)}{\left(\frac{\lambda \alpha}{(\alpha+\mu)^{2}}+1\right)\left(\frac{\lambda \alpha}{(\alpha+\mu)^{2}}+2\right)} \\
E\left(X^{2}\right)=\frac{\eta}{(\eta+\alpha)} \frac{6\left(\frac{\lambda}{(\alpha+\mu)}\right)^{2}{ }_{1} F_{1}\left(4 ; \frac{\lambda \alpha}{(\alpha+\mu)^{2}}+4 ; \frac{\lambda \alpha}{(\alpha+\mu)^{2}}\right)}{\left(\frac{\lambda \alpha}{(\alpha+\mu)^{2}}+1\right)\left(\frac{\lambda \alpha}{(\alpha+\mu)^{2}}+2\right)\left(\frac{\lambda \alpha}{(\alpha+\mu)^{2}}+3\right)} .
\end{gathered}
$$

Similarly, the higher factorial moments can be obtained from (4.7) by successive differentiation with respect to $z$ and evaluating at $z=1$. 
It can be seen that

$\mathrm{P}$ (Server is busy)

$$
\begin{aligned}
=\sum_{n=1}^{\infty} P_{n} & =(1-Q) \sum_{n=1}^{\infty}(n+1)\left(\frac{\lambda}{\alpha+\mu}\right)^{n} \\
& \times \frac{{ }_{1} F_{1}\left(n+2 ; \frac{\lambda \alpha}{(\alpha+\mu)^{2}}+n+2 ; \frac{-\lambda \mu}{(\alpha+\mu)^{2}}\right)}{\left(\frac{\lambda \alpha}{(\alpha+\mu)^{2}}+1\right)\left(\frac{\lambda \alpha}{(\alpha+\mu)^{2}}+2\right) \ldots\left(\frac{\lambda \alpha}{(\alpha+\mu)^{2}}+n+1\right)}
\end{aligned}
$$

$\mathrm{P}($ Server is idle or under repair $)=Q+(1-Q) \frac{{ }_{1} F_{1}\left(2 ; \frac{\lambda \alpha}{(\alpha+\mu)^{2}}+2 ; \frac{-\lambda \mu}{(\alpha+\mu)^{2}}\right)}{\frac{\lambda \alpha}{(\alpha+\mu)^{2}}+1}$ $\mathrm{P}$ (Server is busy \Server is up)

$$
\begin{aligned}
& =\sum_{n=1}^{\infty}(n+1)\left(\frac{\lambda}{\alpha+\mu}\right)^{n} \\
& \times \frac{{ }_{1} F_{1}\left(n+2 ; \frac{\lambda \alpha}{(\alpha+\mu)^{2}}+n+2 ; \frac{-\lambda \mu}{(\alpha+\mu)^{2}}\right)}{\left(\frac{\lambda \alpha}{(\alpha+\mu)^{2}}+1\right)\left(\frac{\lambda \alpha}{(\alpha+\mu)^{2}}+2\right) \ldots\left(\frac{\lambda \alpha}{(\alpha+\mu)^{2}}+n+1\right)},
\end{aligned}
$$

$\mathrm{P}($ Server is idle $\backslash$ Server is up $)=\frac{1 F_{1}\left(2 ; \frac{\lambda \alpha}{(\alpha+\mu)^{2}}+2 ; \frac{-\lambda \mu}{(\alpha+\mu)^{2}}\right)}{\frac{\lambda \alpha}{(\alpha+\mu)^{2}}+1}$.

\section{System Reliability and Availability Analysis}

The reliability of a system is defined as the probability that the system will operate adequately over a given period of time, subject to the given environmental conditions. For example, in computer systems no matter how reliable the components of the system, there is still a chance that it will fail. In this section, the reliability indices like the system availability, system reliability and mean time to failure for the model under consideration are discussed.

In systems with repair, the metric for system performance is the system availability $A(t) . A(t)$ denote the probability that the system is providing service or the server is idle at time t. Then

$$
A(t)=1-Q(t)=\frac{\eta}{\eta+\alpha}+\frac{\alpha}{\eta+\alpha} e^{-(\eta+\alpha) t} .
$$

The average availability of the system in the interval $[0, t]$ is

$$
\overline{A(t)}=\frac{1}{t} \int_{0}^{t} A(u) d u=\frac{\eta}{\eta+\alpha}+\frac{\alpha}{(\eta+\alpha)^{2}} \frac{1-e^{-(\eta+\alpha) t}}{t}
$$


Similarly the reliability function $R(t)$, the mean time to system failure $M T T F$, the mean time to repair $M T T R$ and the maintability $M(t)$ of the system can be obtained directly as in [8].

\section{Appendix}

The confluent hypergeometric function, also referred to as Kummer function, is defined by

$$
{ }_{1} F_{1}(a, c, z)=1+\frac{a}{c} \cdot \frac{z}{1 !}+\frac{a(a+1)}{c(c+1)} \frac{z^{2}}{2 !}+\ldots=\sum_{k=0}^{\infty} \frac{(a)_{k} z^{k}}{(c)_{k} k !}
$$

for $z \in C$, parameters $a, c \in C$ ( $c$ not a negative integer), with $(\alpha)_{n}$, known as Pochhammer symbol, defined by

$$
(\alpha)_{n}=\frac{\Gamma(\alpha+1)}{\Gamma(\alpha-n+1)}, \quad n=0,1,2, \ldots
$$

We observe that

$$
{ }_{1} F_{1}(0 ; c ; z)=1 .
$$

The recurrence relation for the confluent hypergeometric function is given by

$$
c(c-1)_{1} F_{1}(a-1, c-1, z)-a z_{1} F_{1}(a+1 ; c+1 ; z)=c(c-1-z){ }_{1} F_{1}(a, c, z)
$$

(see Abramowitz and Stegun [1]).

The following identities are obtained form Lorentzen and Waadeland [10] and Andrews [2]:

$$
\frac{{ }_{1} F_{1}(a+1 ; c+1 ; z)}{{ }_{1} F_{1}(a ; c ; z)}=\frac{c}{c-z+} \quad \frac{(a+1) z}{c-z+1+} \quad \frac{(a+2) z}{c-z+2+} \quad \ldots
$$

which can be rewritten as

$$
\begin{gathered}
c \frac{{ }_{1} F_{1}(a ; c ; z)}{{ }_{1} F_{1}(a+1 ; c+1 ; z)}-\quad(c-z)=\frac{(a+1) z}{c-z+1+} \frac{(a+2) z}{c-z+2+} \ldots, \\
\sum_{k=0}^{\infty} \frac{(a)_{k} y^{k}}{(c)_{k} k !}{ }_{1} F_{1}(a+k ; c+k ; x)={ }_{1} F_{1}(a ; c ; x+y)
\end{gathered}
$$

and

$$
\frac{\partial^{n}}{\partial z^{n}}{ }_{1} F_{1}(a, c ; z)=\frac{(a)_{n}}{(c)_{n}}{ }_{1} F_{1}(a+n ; c+n ; z)
$$




\section{Acknowledgements}

The author sincerely thanks the management and the principal, SSN Institutions for their constant support towards the successful completion of this work.

\section{References}

[1] M. Abramowitz and I. Stegun, Handbook of Mathematical Functions, Dover, New York (1965).

[2] L.C. Andrews, Special Functions of Mathematics for Engineers, (2nd edition), McGrawHill, Singapore (1992).

[3] N.T.J. Bailey, A continuous time treatment of a single queue using generating functions, J. Royal Stat. Soc. Series B, 16, (1954), 288-291.

[4] D. G. Champernowne, An elementary method of solution of the queueing problem with a single server and constant parameters, J. Royal Stat. Soc. Series B, 18, No. 1 (1956), $125-128$.

[5] B.W. Conolly, A difference equation technique applied to the simple queue with arbitrary arrival interval distribution, J. Royal Stat. Soc. Series B, 20, No. 1 (1958), 268-275.

[6] E. Gelenbe, Product form queueing networks with positive and negative customers, $J$. App. Probab, 28, No. 3 (1991), 656-663.

[7] D. Gross and C.M. Harris, Fundamentals of queueing theory, Wiley, New york (1985).

[8] B. Krishna Kumar and S. Pavai Madheswari, Transient analysis of an M/M/1 queue subject to catastrophes and server failures, Stoch. Anal. Appl. 23, No. 2 (2005) 329-340.

[9] B. Krishna Kumar, A. Vijayakumar, and S. Sophia, Transient analysis of a Markovian queue with chain sequence rates and total catastrophes, Int. J. Oper. Res., 5, No. 4 (2009), 375-391.

[10] L. Lorentzen and H. Waadeland, Continued Fractions with Application, North-Holland, Amsterdam (1992).

[11] R. Nagarajan and J. Kurose, On defininig, computing and guaranteeing quality-of-service in high-speed networks, In: Proc. of INFOCOM92., Florence, Italy, (1992), 2016-2025.

[12] B. Natvig, On the transient state probabilities for a queueing model where potential customers are discouraged by queue length, J. Appl. Probab., 11, No. 2 (1974), 345-354.

[13] Ng Chee Hock, Queueing Modelling Fundamentals, John Wiley, Chichester (1996).

[14] P.R. Parthasarathy and N. Selvaraju, Transient analysis of a queue where potential customers are discouraged by queue length, Math. Prob. in Engrg, 7 No. 5 (2001), 433454.

[15] P.R. Parthasarathy and R. Sudhesh, The overflow process from a state-dependent queue, Int. J. Comp. Math, 82, No. 9 (2005), 1073-1093.

[16] P.R. Parthasarathy and R. Sudhesh, Time dependent analysis of a single server retrial queue with state dependent rates, Oper. Res. Lett, 35, No. 5 (2007), 601-611.

[17] P.R.Parthasarathy and K.V. Vijayashree, Fluid queues driven by a discouraged arrivals queue, Int. J. Math. Math. Sci., 2003, No. 24 (2003), 1509-1528. 
[18] N.Paz and U. Yechiali, An M/M/1 queue in random environment with disasters, AsiaPacific J. Oper. Res., 31, No. 3 (2014), 1-12.

[19] S. Sophia and A. Vijayakumar, Exact solution to tandem fluid queues with controlled input. Stoch. Anal. Appl., 33, No. 1 (2015), 21-39.

[20] A.M.K. Tarabia, Transient and steady state analysis of an M/M/1 queue with balking, catastrophes, server failures and repairs, J. Ind. Manage. Optim., 7, No. 4 (2011), 811823.

[21] D. Towsley and S.K. Tripathi, A single server priority queue with server failures and queue flushing, Oper. Res. Lett., 10, No. 6 (1991), 353-362.

[22] H.S. Wall, Analytical Theory of Continued Fractions, D.Van.Nostrand, Toronto (1948).

[23] P. Wartenhorst, N-Parallel queueing systems with server breakdown and repair, NASA Tech. Repo., Amsterdam:CWI, N94-21232, (1992),1-35. 